OPEN ACCESS

Edited by:

Penghua Wang,

New York Medical College,

United States

Reviewed by:

Jianfeng Dai,

Soochow University, China

Fengwei Bai

University of Southern Mississippi,

United States

Manoj N. Krishnan,

National University of Singapore,

Singapore

*Correspondence:

Chunxia Jing

jcxphd@gmail.com

Guang Yang

guangyangphd@gmail.com

Received: 25 April 2017 Accepted: 27 June 2017 Published: 12 July 2017

Citation:

Guo C, Zhou Z, Wen Z, Liu Y, Zeng C, Xiao D, Ou M, Han Y, Huang S, Liu D, Ye $X$, Zou $X$, Wu J, Wang $H$, Zeng EY,

Jing $C$ and Yang $G$ (2017) Global Epidemiology of Dengue Outbreaks in 1990-2015: A Systematic Review and

Meta-Analysis.

Front. Cell. Infect. Microbiol. 7:317. doi: 10.3389/fcimb.2017.00317

\section{Global Epidemiology of Dengue Outbreaks in 1990-2015: A Systematic Review and Meta-Analysis}

\author{
Congcong Guo ${ }^{1}$, Zixing Zhou ${ }^{1}$, Zihao Wen ${ }^{1}$, Yumei Liu ${ }^{1,2}$, Chengli Zeng ${ }^{1}$, Di Xiao', \\ Meiling Ou ${ }^{1,2}$, Yajing Han ${ }^{1,2}$, Shiqi Huang ${ }^{1}$, Dandan Liu ${ }^{1}$, Xiaohong Ye ${ }^{1,2}$, Xiaoqian Zou ${ }^{1,2}$, \\ Jing Wu ${ }^{1,2}$, Huanyu Wang ${ }^{3,4}$, Eddy Y. Zeng ${ }^{5}$, Chunxia Jing ${ }^{1,5 *}$ and Guang Yang ${ }^{2,5 *}$ \\ ${ }^{1}$ Department of Epidemiology, School of Medicine, Jinan University, Guangzhou, China, ${ }^{2}$ Department of Parasitology, School \\ of Medicine, Jinan University, Guangzhou, China, ${ }^{3}$ Department of Viral Encephalitis, Chinese Center for Disease Control and \\ Prevention, Institute for Viral Disease Control and Prevention, National Institute for Viral Disease Control and Prevention, \\ Beijing, China, ${ }^{4}$ State Key Laboratory for Infectious Disease Prevention and Control, Chinese Center for Disease Control and \\ Prevention, Beijing, China, ${ }^{5}$ Guangzhou Key Laboratory of Environmental Exposure and Health, Guangdong Key Laboratory \\ of Environmental Pollution and Health, School of Environment, Jinan University, Guangzhou, China
}

Dengue is an arthropod-borne infectious disease caused by dengue virus (DENV) infection and transmitted by Aedes mosquitoes. Approximately 50-100 million people are infected with DENV each year, resulting in a high economic burden on both governments and individuals. Here, we conducted a systematic review and meta-analysis to summarize information regarding the epidemiology, clinical characteristics, and serotype distribution and risk factors for global dengue outbreaks occurring from 1990 to 2015. We searched the PubMed, Embase and Web of Science databases through December 2016 using the term "dengue outbreak." In total, 3,853 studies were identified, of which 243 studies describing 262 dengue outbreaks met our inclusion criteria. The majority of outbreak-associated dengue cases were reported in the Western Pacific Region, particularly after the year 2010; these cases were primarily identified in China, Singapore and Malaysia. The pooled mean age of dengue-infected individuals was 30.1 years; of the included patients, $54.5 \%$ were male, $23.2 \%$ had DHF, $62.0 \%$ had secondary infections, and $1.3 \%$ died. The mean age of dengue patients reported after 2010 was older than that of patients reported before 2010 (34.0 vs. 27.2 years); however, the proportions of patients who had DHF, had secondary infections and died significantly decreased after 2010. Fever, malaise, headache, and asthenia were the most frequently reported clinical symptoms and signs among dengue patients. In addition, among the identified clinical symptoms and signs, positive tourniquet test $(O R=4.86)$, ascites $(O R=13.91)$ and shock (OR $=308.09$ ) were identified as the best predictors of dengue infection, DHF and mortality, respectively (both $P<0.05$ ). The main risk factors for dengue infection, $\mathrm{DHF}$ and mortality were living with uncovered water container $(O R=1.65)$, suffering from 
hypotension $(O R=6.18)$ and suffering from diabetes mellitus $(O R=2.53)$, respectively (all $P<0.05$ ). The serotype distribution varied with time and across $\mathrm{WHO}$ regions. Overall, co-infections were reported in $47.7 \%$ of the evaluated outbreaks, and the highest pooled mortality rate (2.0\%) was identified in DENV-2 dominated outbreaks. Our study emphasizes the necessity of implementing programs focused on targeted prevention, early identification, and effective treatment.

Keywords: dengue, outbreak, epidemiology, clinical characteristic, risk factor, serotype, systematic review, meat-analysis

\section{INTRODUCTION}

Only 9 countries had experienced severe dengue epidemics before 1970; however, at present, dengue fever has affected more than 100 countries in tropical and subtropical regions (Organization, 2016). It was estimated by WHO that 50-100 million dengue infections occur annually, with a 30-fold increase in global incidence observed over the past 50 years (WHO, 2012). Today, dengue virus (DENV) poses a major threat to global public health, and approximately two-fifths of the world's population is at risk of dengue infection (Lancet, 2013; Screaton et al., 2015).

Since the first dengue outbreak was reported in 1779 in Jakarta, Indonesia (Wu et al., 2011), this disease has become a public health threat that is associated with remarkable morbidity and mortality. The case fatality rate in untreated dengue patients has been reported to reach 20\%, but this rate can be reduced to less than $1 \%$ under expert clinical management and with careful fluid replacement (Simmons et al., 2012). The number of dengue outbreaks caused by the four dengue virus serotypes (DENV-1 to DENV-4) has increased since 1980, mainly affecting Asia, South America and the Caribbean (Jansen and Beebe, 2010; Amarasinghe et al., 2011). The various serotypes of the DENV are transmitted predominantly by the mosquito vector, Aedes aegypti (Guzman and Harris, 2015); however, DENV can also be transmitted by other species of the genus Aedes, including Aedes albopictus (Barcelos, 2014). While most dengue patients recover after experiencing self-limiting illness, a small proportion progress to dengue hemorrhagic fever (DHF) (WHO/TDR, 2009). DHF may be classified into four severity grades, with grades III and IV being defined as dengue shock syndrome (DSS) (WHO/TDR, 2009). Although the characteristics of dengue infection have been well described, previous studies have mainly focused on the descriptions of single outbreaks, and few of studies have concentrated on systematically summarizing the epidemiological characteristics of dengue outbreaks worldwide. In this situation, it may difficult to keep abreast of the trends of global dengue outbreaks.

At present, little attention has been specifically paid to summarizing global dengue outbreaks. We performed a robust systematic analysis of all available data to gain a better understanding of the global epidemiology, clinical manifestations, and serotype distribution and risk factors for dengue outbreaks.

\section{MATERIALS AND METHODS}

\section{Searching Strategy and Selection Criteria}

We searched the PubMed, Embase and Web of Science databases to identify articles describing dengue outbreaks that occurred between Jan 1, 1990 to Dec 1, 2016 (Figure 1). The following search terms were used as a text word in each database: PubMed, "dengue outbreak" in all fields; limited to human studies; Embase, "dengue outbreak" in all fields; limited to human studies; and Web of Science, "dengue outbreak" in the topic field with the exclusion of veterinary studies. Two independent reviewers (C.C.G and Z.X.Z) screened the titles and abstracts of all related manuscripts, searched reference lists of the identified studies and obtained full texts for potentially relevant articles. Studies without any dengue outbreak data available, that described outbreaks including less than 5 dengue patients or that did not focus on a specific dengue outbreak were excluded. When different articles described the same outbreak but provided different data, all studies were included.

\section{Data Extraction}

The following data were independently extracted from each included study by two investigators (C.C.G and Z.X.Z): author, year of publication, year of outbreak, WHO region, country, city, mosquito species, patient characteristics (including demographic characteristics, risk factors, clinical symptoms and number of secondary cases), dengue serotype, number of patients (including DF, DHF, and DSS patients), and number of deaths. Any disagreements were adjudicated by a senior investigator (C.X.J).

\section{Analysis}

We described the epidemiology, clinical manifestations, risk factors and serotype distribution using the number of studies and cases, which are expressed as proportions with 95\% CIs for categorical variables (sex, dengue case classification, primary or secondary infection, signs, symptoms and mortality) and means and corresponding 95\% CIs for continuous variables (age). We compared the epidemiologic characteristics of dengue outbreaks occurring before 2010 and after 2010 (including 2010) using two-proportion z-tests for categorical variables and Student's $t$ tests for continuous variables. Meta-analyses were performed using Comprehensive Meta-Analysis version 2.2.064 software (Biostat Inc., NJ, USA). We assessed the level of heterogeneity across studies using $P_{h}$ and $I^{2}$ (Higgins and Thompson, 2002). If a $P_{h}$-value was greater than 0.10 , a fixed-effects model was used; otherwise, a random-effects model was selected (Handoll, 


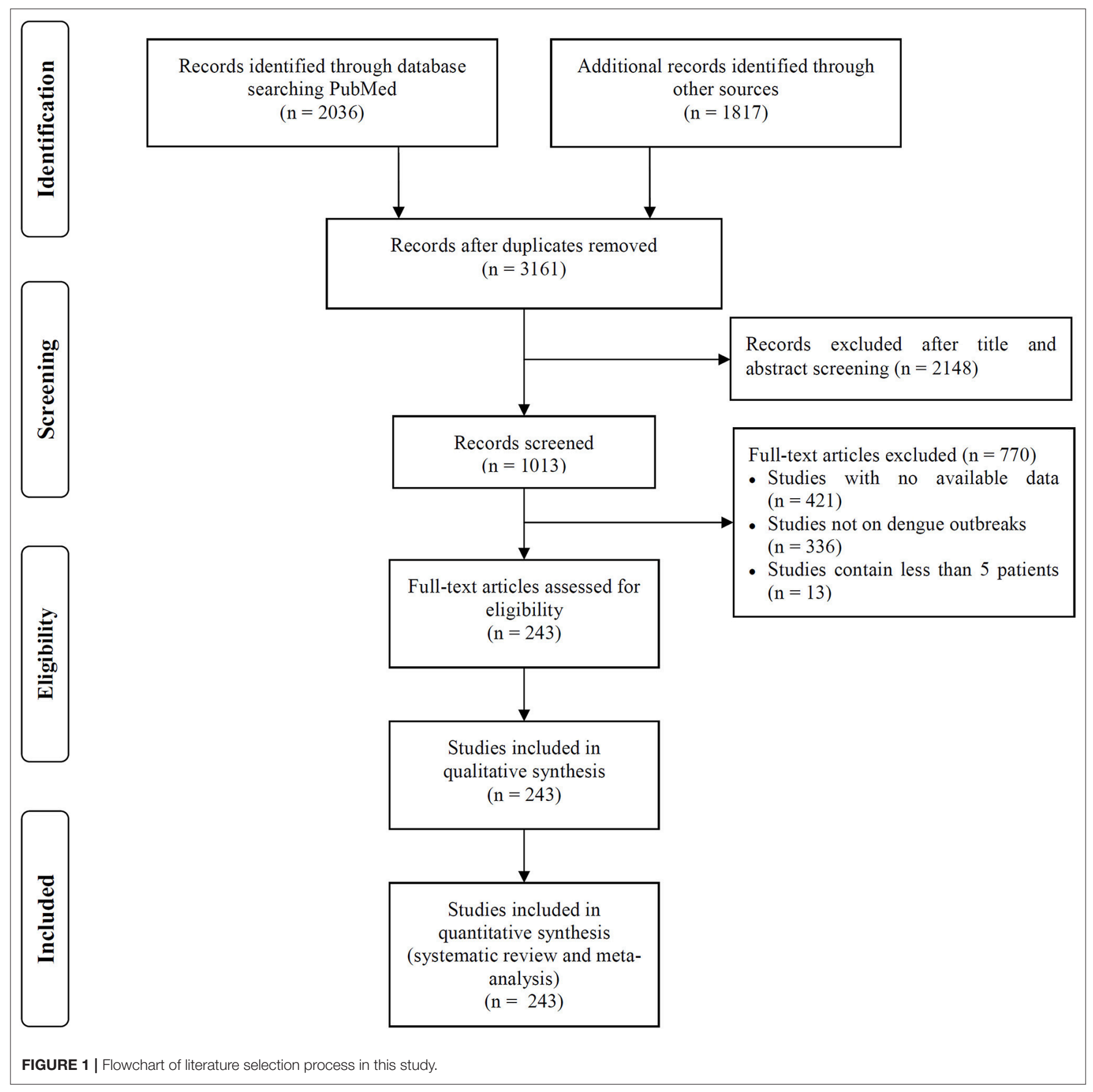

2006). The meta-analysis corresponded with recommendations from the Preferred Reporting Items for Systematic Reviews and Meta-Analyses (PRISMA) statement (Supplementary File S2). Medians (ranges) were converted to means (SDs) using previously proposed formulas (Hozo et al., 2005). Distribution maps for global dengue outbreaks and serotypes were generated in R software, version 3.3.1 (R Core Team, Vienna, Austria), using ggplot2, maps, mapproj and sp packages. The correlations between the rates of mortality, DHF, and secondary infections were assessed by generating Spearman correlation coefficients.

\section{RESULTS}

\section{Systematic Review}

We included 243 articles describing 262 dengue outbreaks that occurred between 1990 and 2015 (Figure 1, Supplementary Table 1, Supplementary File S1). One hundred and twelve outbreaks described in 104 articles occurred after 2010. Among countries worldwide, the highest numbers of outbreak (58/262) are observed in India, followed by China (38/262) and Brazil (24/262) from 1990 to 2015. All the outbreaks occurred in 
tropical (77/262) and subtropical (174/262) regions, except for one outbreak, which occurred in Nîmes, 2015 (Succo et al., 2016), and was considered the first considerable dengue outbreak in mainland France (Figure 2). Among the six WHO regions, the largest number of outbreaks occurred in the Southeast Asia region (82/262), followed by the Western Pacific region (72/262) and the American region (65/262), accounting for more than $83.6 \%$ of outbreaks overall. The European region $(6 / 262)$ was least affected by dengue outbreaks, with only 4 outbreaks reported in France (three in overseas departments and regions of France and one in mainland France) and 2 outbreaks reported in Portugal. However, the Western Pacific Region had most dengue outbreaks reported after 2010 (Western Pacific: 33/112 > Southeast Asia: 27/112 > Americas: 24/112 > Eastern Mediterranean: 13/112 > Africa: 11/112 > Europe: 4/112).

\section{Epidemiology}

By the end of 2016, a total of 291,964 outbreak-associated dengue cases had been reported in the literature, mainly from China (27.9\%), Singapore $(27.0 \%)$ and Malaysia (15.1\%). The majority (72.4\%) of dengue patients were reported in the Western Pacific region, followed by the American region (19.4\%), Southeast Asia Region (4.8\%), Eastern Mediterranean region (1.5\%), European region (1.5\%) and African region (0.3\%). Outbreaks occurring before 2010 (Americas $>$ Western Pacific > South East Asia > Eastern Mediterranean $>$ Europe $>$ Africa) accounted for $28.6 \%$ of the total number of cases, the majority of which were reported in Cuba (28.1\%), Singapore (19.3\%) and Puerto Rico (13.4\%), while patients in outbreaks after 2010 (Western Pacific > Americas $>$ South East Asia $>$ Europe $>$ Eastern Mediterranean
$>$ Africa) were mainly from China (36.8\%), Singapore (30.1\%), and Malaysia (20.9\%). 50.0\% outbreaks occurred in urban areas (21/42), $28.6 \%$ in rural areas (12/42) and $21.4 \%$ in both urban and rural areas (9/42). It is important to note that nearly all rural outbreaks occurred after 2000 (except one in Malaysia, 1999; Cheah et al., 2006). It is consistent with the view that dengue once confined to urban areas has penetrated into the rural setup (Ishak et al., 1997). The improved road systems, better socio-economic situations and established agricultural settlements in rural areas may increase the Ae. albopictus population, and thus spread of rural dengue fever among the rural communities (Chang et al., 1997).

The pooled mean age of the patients was 30.1 years, and $54.5 \%$ were male (Table 1, Supplementary Table 2). The results of the meta-analyses indicated there to be significant associations between dengue infection and two variables: male gender (OR: 1.10, 95\% CI: 1.01-1.20) and living with uncovered water container (OR: 1.65, 95\% CI: 1.15-2.37) (Figure 3, Supplementary Table 3). The pooled rate of DHF was 23.2\%; secondary infection (OR: 1.86, 95\% CI: 1.46-2.37), diabetes mellitus (OR: 2.31, 95\% CI: 1.58-3.38), hypotension (OR: 6.18, 95\% CI: 1.61-23.71) and renal insufficiency (OR: 5.26, 95\% CI: 1.77-15.64) were at increased odds of DHF (Figure 3, Supplementary Table 4). The rate of secondary infections among dengue patients was $62.0 \%$ in the meta-analysis of the 35 studies reporting these data; patients with a platelet count $<100 \times 10^{9} / \mathrm{L}$ had increased odds of secondary infection (OR: 4.11, 95\% CI: 1.64-10.31) (Supplementary Table 5). In addition, the pooled rate of mortality derived from the 72 studies reporting this outcome was $1.3 \%$; patients with underlying diseases, such as diabetes

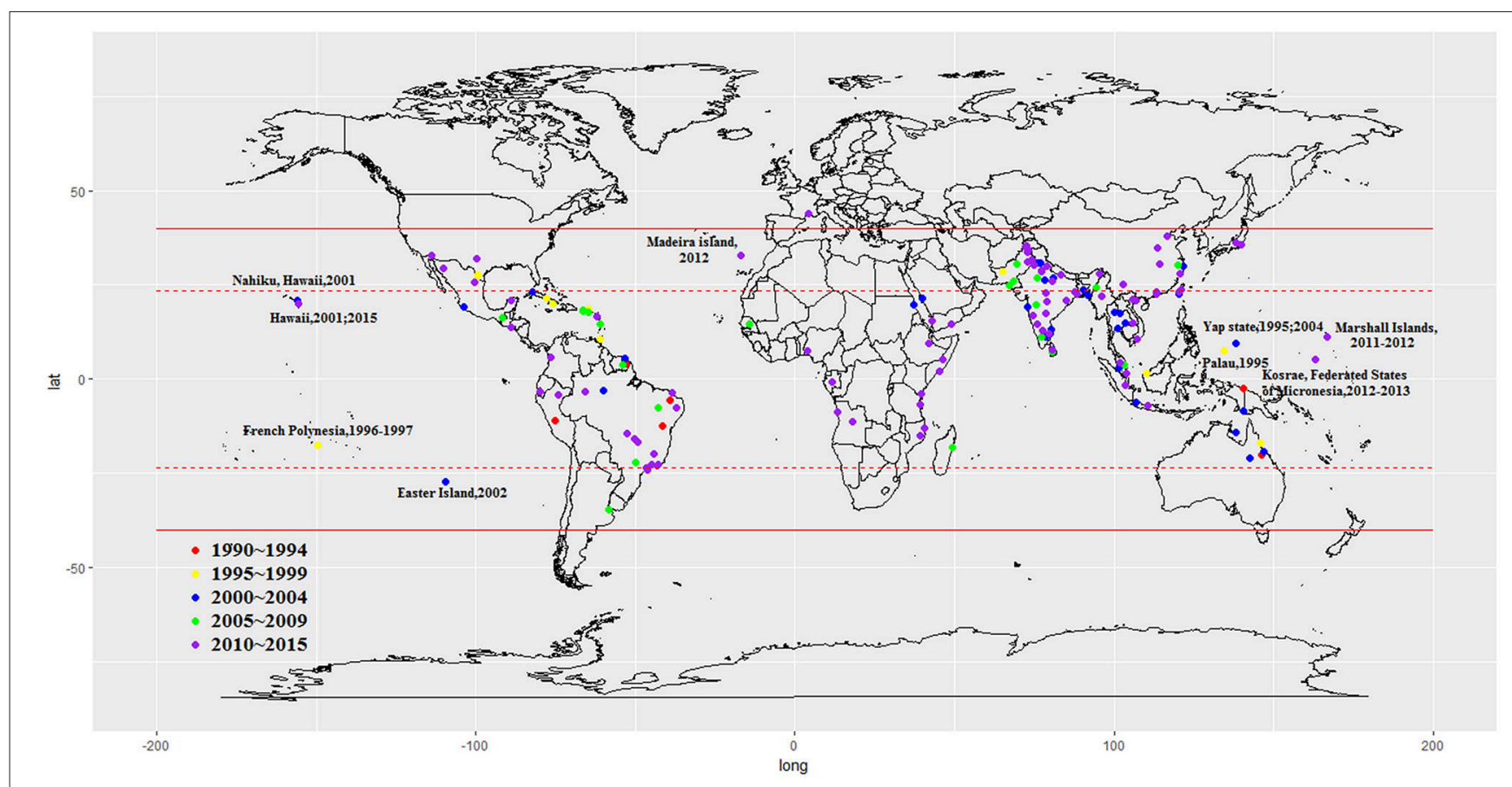

FIGURE 2 | Global dengue outbreaks distribution from 1990-2015. 
mellitus (OR: 2.53, 95\% CI: 1.51-4.24) and hypertension (OR: 2.36, 95\% CI: 1.37-4.07) were at increased odds of mortality (Figure 3, Supplementary Table 6).

Patient age distributions, DHF rates, secondary infection rates and mortality rates differed significantly between outbreaks occurring before the year 2010 and occurring after 2010 (Table 1). Dengue patients identified in association with outbreaks occurring after 2010 were significantly older than those identified in association with outbreaks occurring before 2010 (mean, 95\% CI: 34.0 , 30.1-38.0 vs. 27.2 , 24.5-30.0; $P=0.006$ ). The rates of DHF, secondary infection and mortality significantly decreased after 2010 (all $P<0.001$ ). No significant gender difference was identified between these two time periods $(P=$

TABLE 1 | Epidemiologic factors of dengue patients in global outbreaks.

\begin{tabular}{lccc}
\hline Variables & $\begin{array}{c}\text { No. studies } \\
\text { meta-analyzed }\end{array}$ & $\begin{array}{c}\text { Meta-analysis, pooled } \\
\text { data (95\% Cl)* }\end{array}$ & P value \\
\hline Mean age & 96 & $30.1(27.7-32.5)$ & 0.006 \\
Before 2010 & 55 & $27.2(24.5-30.0)$ & \\
After 2010 & 41 & $34.0(30.1-38.0)$ & \\
Male (\%) & 146 & $54.5(53.2-55.7)$ & 0.194 \\
Before 2010 & 83 & $54.7(52.6-56.7)$ & \\
After 2010 & 63 & $54.3(52.5-56.0)$ & \\
DHF (\%) & 107 & $23.2(18.3-29.0)$ & $<0.001$ \\
Before 2010 & 85 & $18.8(14.3-24.3)$ & \\
After 2010 & 22 & $15.7(7.6-29.6)$ & \\
Secondary infection (\%) & 35 & $62.0(53.5-69.8)$ & $<0.001$ \\
Before 2010 & 20 & $66.7(55.1-76.6)$ & \\
After 2010 & 15 & $55.9(43.8-67.3)$ & \\
Fatal cases (\%) & 72 & $1.3(0.9-2.0)$ & $<0.001$ \\
Before 2010 & 46 & $1.9(1.2-2.9)$ & \\
After 2010 & 26 & $0.7(0.3-1.8)$ & \\
\hline
\end{tabular}

${ }^{\star}$ Random-effects model unless otherwise specified.

${ }^{a}$ Removed articles contained all DHF patients; P-value, two-proportion z-test for categorical factors and Student's t-test for continuous factors.
0.194). The rate of DHF and rate of mortality observed in the included dengue outbreaks were significantly correlated, with an overall Spearman correlation coefficient of $0.64(P<0.001)$. However, no significant correlation was observed between the rate of secondary infection and the rate of mortality $(P=0.223)$.

\section{Clinical Symptoms and Signs}

Fever was the most commonly observed clinical symptom or sign (pooled proportion: 98.1, 95\% CI: 97.2-98.7\%), followed by malaise (76.0, 95\% CI: 64.1-84.9\%), headache $(75.7,95 \% \mathrm{CI}$ : 69.5-81.0\%) and asthenia (74.3, 95\% CI: 45.8-90.8\%), which are listed in Table 2 (Supplementary Table 7). Hemorrhagic manifestations were observed in $25.8 \%$ (95\% CI: $21.0-31.1 \%$ ) of the patients, of which petechiae $(22.3,95 \% \mathrm{CI}: 16.5-29.3 \%)$ was the most common.

The rates of myalgia, chill, rash, eye/retro-orbital pain, petechiae, exanthema, lethargy, lymphadenopathy, thrombocytopenia, leukopenia, conjunctival injection, and positive tourniquet test results were significantly greater in the dengue-infected group than the laboratory negative group (all $P<0.05)$. On the other hand, since most of the laboratory negative patients were infected with influenza, those patients more frequently suffered from sore throat, nasal congestion and cough than did dengue-infected patients (all $P<0.05$ ) (Figure 4, Supplementary Table 3).

Blood manifestations (OR: 9.57, 95\% CI: 4.78-19.15), pleural effusion (OR: 12.44, 95\% CI: 7.07-21.91), ascites (OR: 13.91, 95\% CI: 8.03-24.11) and morality (OR: 11.46 , 95\% CI: 4.1631.56) were more common in DHF patients than DF patients, and other symptom and sign comparisons are shown in Figure 4, Supplementary Table 4. However, body-ache (OR: 0.60, 95\% CI: $0.36-0.99$ ) was identified significantly less frequently in $\mathrm{DHF}$ patients than DF patients.

Patients with shock symptom were at significantly increased odds of mortality (OR: 308.09, 95\% CI: 42.56-2230.53). In contrast, headaches were negatively associated with mortality (OR: 0.46, 95\% CI: 0.22-0.95) (Figure 4, Supplementary Table 6).

\begin{tabular}{|c|c|c|c|c|c|}
\hline & $\begin{array}{l}\text { Odds } \\
\text { ratio }\end{array}$ & $\begin{array}{l}\text { Lower } \\
\text { limit }\end{array}$ & $\begin{array}{l}\text { Upper } \\
\text { limit }\end{array}$ & Z-Value & p-Valt \\
\hline \multicolumn{6}{|l|}{ Patients $=$ Dengue Infection } \\
\hline Gender (male) & 1.10 & 1.01 & 1.20 & 2.17 & 0.03 \\
\hline Uncovered water container & 1.65 & 1.15 & 2.37 & 2.71 & 0.00 \\
\hline \multicolumn{6}{|c|}{ Patients $=$ Dengue Hemorrhagic Fever } \\
\hline Diabetes mellitus & 2.31 & 1.58 & 3.38 & 4.32 & 0.0 \\
\hline Hypotension & 6.18 & 1.61 & 23.71 & 2.66 & 0.00 \\
\hline Renal insufficiency & 5.26 & 1.77 & 15.64 & 2.99 & 0.0 \\
\hline Secondary infection & 1.86 & 1.46 & 2.37 & 4.99 & 0.0 \\
\hline \multicolumn{6}{|l|}{ Patients $=$ Death } \\
\hline Diabetes mellitus & 2.53 & 1.51 & 4.24 & 3.52 & 0.0 \\
\hline Hypertension & 2.36 & 1.37 & 4.07 & 3.08 & 0.0 \\
\hline
\end{tabular}

FIGURE 3 | Meta-analysis: forest plot of the associations between risk factors and dengue infection, DHF and mortality in dengue outbreaks. 
TABLE 2 | Clinical symptoms and signs of patients in dengue outbreaks.

\begin{tabular}{|c|c|c|}
\hline Variables & $\begin{array}{c}\text { No. studies } \\
\text { meta-analyzed }\end{array}$ & $\begin{array}{l}\text { Meta-analysis, pooled } \\
\text { data }(95 \% \mathrm{Cl})^{*}\end{array}$ \\
\hline \multicolumn{3}{|l|}{ CONSTITUTIONAL } \\
\hline Fever & 88 & $98.1(97.2-98.7)$ \\
\hline Chills & 14 & $65.3(58.3-71.6)$ \\
\hline Myalgia & 65 & $64.2(58.1-69.8)$ \\
\hline Arthralgia & 53 & $53.6(46.0-61.0)$ \\
\hline Lethargy & 5 & $67.1(32.6-89.6)$ \\
\hline Malaise & 9 & $76.0(64.1-84.9)$ \\
\hline Asthenia & 6 & $74.3(45.8-90.8)$ \\
\hline Body-ache & 13 & $67.2(55.2-77.3)$ \\
\hline Back pain & 9 & $57.3(32.2-79.1)$ \\
\hline Sore throat & 12 & $19.7(13.4-28.1)$ \\
\hline Eye pain & 6 & $27.8(13.7-48.1)$ \\
\hline Retro-orbital pain & 38 & $35.1(27.0-44.2)$ \\
\hline Lymphadenopathy & 13 & $9.2(4.4-18.2)$ \\
\hline \multicolumn{3}{|l|}{ GASTROINTESTINAL } \\
\hline Vomiting & 36 & $39.8(35.0-44.9)$ \\
\hline Nausea & 22 & $42.0(34.0-50.4)$ \\
\hline Diarrhea & 36 & $20.7(17.3-24.7)$ \\
\hline Anorexia & 17 & $47.8(34.9-61.0)$ \\
\hline Ascites & 25 & $10.2(5.3-18.8)$ \\
\hline Icterus/Jaundice & 13 & $2.8(1.5-5.2)$ \\
\hline Abdominal pain & 61 & $32.4(27.9-37.2)$ \\
\hline Hepatomegaly & 41 & $18.9(12.7-27.1)$ \\
\hline Splenomegaly & 20 & $7.7(5.2-11.3)$ \\
\hline Hepatosplenomegaly & 5 & $17.5(8.3-33.3)$ \\
\hline \multicolumn{3}{|l|}{ MUCOCUTANEOUS } \\
\hline Rash & 83 & $29.6(26.1-33.3)$ \\
\hline Pruritus & 5 & $24.1(19.8-29.0)$ \\
\hline Exanthema & 5 & $33.7(11.2-67.1)$ \\
\hline Itching eruption & 6 & $24.0(18.7-30.2)$ \\
\hline \multicolumn{3}{|l|}{ CARDIORESPIRATORY } \\
\hline Cough & 29 & $22.9(17.8-28.8)$ \\
\hline Pleural effusion & 23 & $8.3(4.5-14.9)$ \\
\hline Myocarditis & 5 & $5.7(1.2-22.5)$ \\
\hline Hypotension & 14 & $12.5(7.7-19.7)$ \\
\hline Respiratory disorders & 13 & $8.7(5.3-13.9)$ \\
\hline \multicolumn{3}{|l|}{ NEUROLOGICAL } \\
\hline Headache & 83 & $75.7(69.5-81.0)$ \\
\hline Dizziness & 9 & $22.8(11.7-39.7)$ \\
\hline Seizure & 7 & $2.7(1.8-3.9)^{\star \star}$ \\
\hline Shock & 12 & $9.5(4.2-20.0)$ \\
\hline Convulsion & 5 & $6.1(2.9-12.5)$ \\
\hline Encephalopathy & 8 & $5.0(1.9-12.4)$ \\
\hline \multicolumn{3}{|l|}{ HEMORRHAGIC MANIFESTATIONS } \\
\hline Gingivorrhagia & 16 & $9.7(6.0-15.2)$ \\
\hline Epistaxis & 25 & $11.8(7.6-17.9)$ \\
\hline Hematuria & 16 & $5.0(3.0-8.1)$ \\
\hline Melena & 13 & $16.9(8.1-31.8)$ \\
\hline Petechiae & 30 & $22.3(16.5-29.3)$ \\
\hline Hematemesis & 18 & $13.4(8.0-21.6)$ \\
\hline Bleeding/Hemorrhagic manifestations & 58 & $25.8(21.0-31.1)$ \\
\hline
\end{tabular}

${ }^{\star}$ Random-effects model unless otherwise specified.

${ }^{* *}$ Fixed-effects model.

\section{Serotype}

Studies of 174 outbreaks that occurred between 1990 and 2015 reported dengue serotype data. The highest number of monoinfection outbreaks were caused by DENV-2 $(36,20.7 \%)$, followed by DENV-1 (29,16.7\%), DENV-3 (19, 10.9\%) and DENV-4 (7, 4.0\%). Coinfection with more than one DENV serotype was reported in $47.7 \%$ of the outbreaks; outbreaks involving all four serotypes were the most common $(25,14.4 \%)$, followed by coinfection with DENV-1 and DENV-2 (16, 9.2\%) and coinfection DENV-1, DENV-2 and DENV-3 (12, 6.9\%).

The longitudinal trends in serotype distribution are shown in Figure 5. Between 1990 and 1994, DENV-2 was most frequently identified serotype in the 6 reported outbreaks $(5 / 6,83.3 \%)$. During 1995-1999 and 2000-2004, DENV-2 monoinfection was the predominant serotype observed in dengue outbreaks (11/20, $55.0 \%$ and $10 / 45,22.2 \%$ ). Coinfection dominated subsequent (2005-2009) outbreaks $(12 / 23,52.2 \%)$, especially co-infection with all four serotypes (4/23, 17.4\%). After 2010, DENV-1 dominated the monoinfection outbreaks $(17 / 34,50.0 \%)$ and coinfection with all four serotypes continued to dominate the coinfection outbreaks $(17 / 46,37.0 \%)$. Different serotypes were predominant in different WHO regions after 2010: DENV-1 and DENV-2 were most common in the African region and American region; DENV-1 was most common in the European region; coinfection with all four serotypes was most common in the Southeast Asia region; DENV-2 and DENV-3 were most frequently observed in the Eastern Mediterranean region, and DENV-1 was predominant in the Western Pacific region.

The highest pooled mortality rate was identified in DENV2 dominated outbreaks at $2.0 \%$ (95\% CI: 0.9-4.2\%), followed by DENV-3 (1.6, 95\% CI: 0.4-6.8\%), DENV-4 (0.7, 95\% CI: $0.0-42.3 \%)$ and DENV-1 (0.3, 95\% CI: 0.1-0.9\%).

\section{DISCUSSION}

Our study presents the epidemiology, clinical characteristics, and serotype distribution and risk factors for global dengue outbreaks in humans that occurred between 1990 and 2015. The majority of the 262 outbreaks occurred in developing countries, including India, China and Brazil. These high numbers might be attributed to the limited laboratory facilities and inadequate control measures in these countries (Cattand et al., 2006). The relatively low socioeconomic status of and high population density and ideal environment for the maintenance of mosquitoes in developing countries may also facilitate the transmission of DENV (Figueiredo, 2007; Saswat et al., 2015). After 2010, the majority of dengue outbreaks and dengue patients were identified in the Western Pacific region, which may be mainly attributed to cases in China, Singapore and Malaysia. In contrast, only a few dengue outbreaks were reported in the Europe region, and these outbreaks involved in only two countries (France and Portugal). The disappearance of A. aegypti from the European Basin between the 1950s and 2005 might be one reason for its low-prevalence of dengue outbreaks (Succo et al., 2016). It is important to note that many dengue outbreaks were influenced by the dengue epidemics 


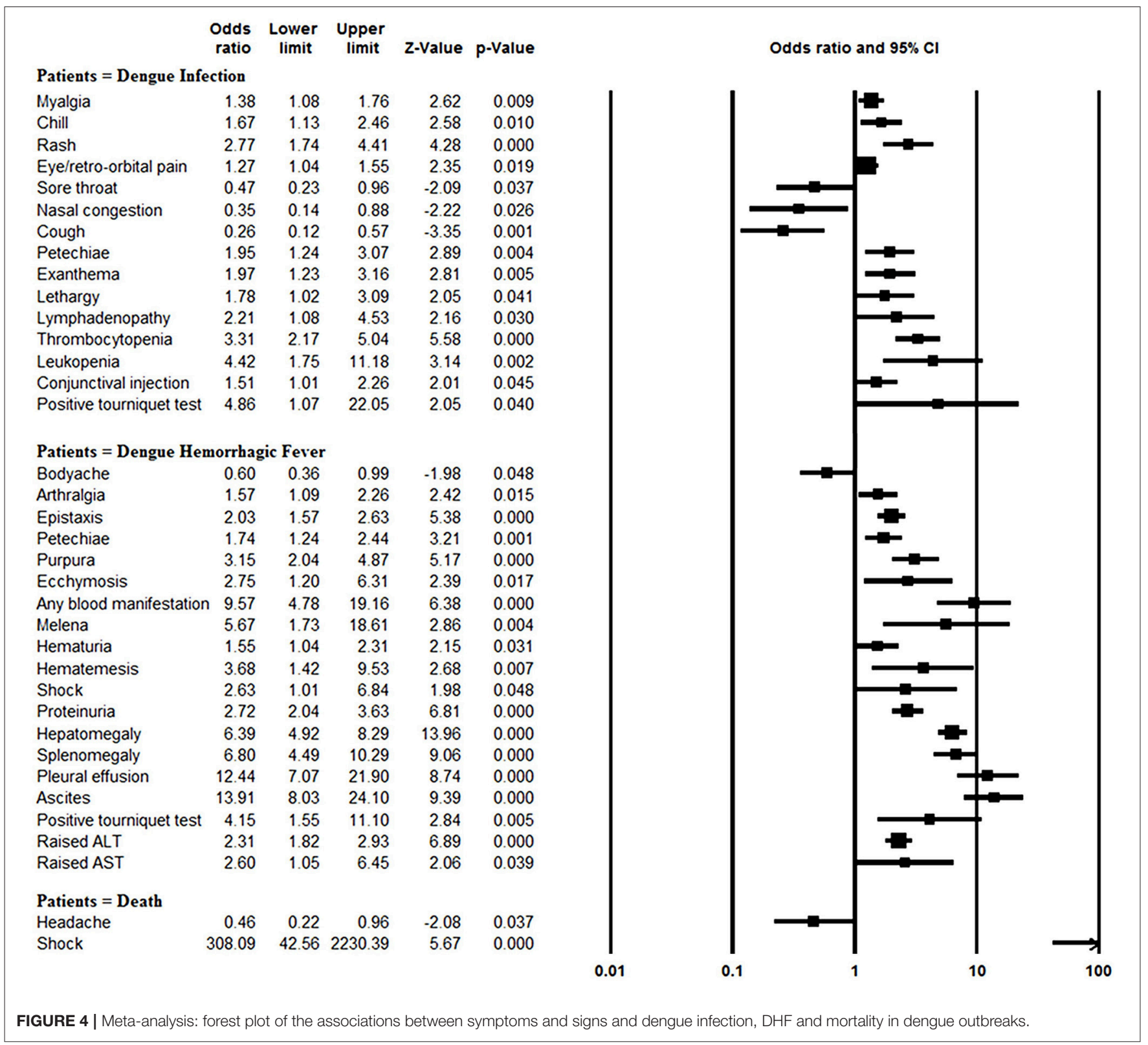

in nearby/neighboring countries. Wang et al. reported that the dengue virus in the Xishuangbanna and Dehong, China outbreak in 2013 was imported from Southeast Asia countries (Wang et al., 2016). Owing to the more frequent interactions between the populations of Southeast Asian countries and China, imported dengue epidemics have been documented in southern China. Additionally, the first major outbreak of dengue in Europe was reported to be most likely imported from Venezuela via travelers to Madeira (Wilder-Smith et al., 2014). Valid laboratory-based disease surveillance system and integrated vector management are required in the border regions of dengue epidemic areas so that future disease outbreaks and spread to other regions can be prevented (Guo et al., 2015).
Ongoing outbreaks likely reflect deficiencies in vector control and prevention. Originating in Africa and spreading to tropical countries in the seventeenth and eighteenth centuries, $A$. aegypti has been considered the primary dengue vector due to its enhanced viral duplication capability, thus increasing the probability of viral transmission (Gubler, 2011; Chepkorir et al., 2014; Whitehorn et al., 2015). Sixty of the articles included in our meta-analysis mentioned mosquitoes. Consistent with prior knowledge, the most prevalent mosquito was A. aegypti (76.7\%), followed by $A$. albopictus (43.3\%). In addition, the pooled mortality rate in outbreaks associated with $A$. aegypti mosquitoes (0.7, 95\% CI: $0.3-1.7 \%)$ was higher than the pooled mortality rate in outbreaks associated with $A$. albopictus mosquitoes $(0.4$, 95\% CI: 0.1-2.5\%). Dengue outbreaks caused by A. albopictus 

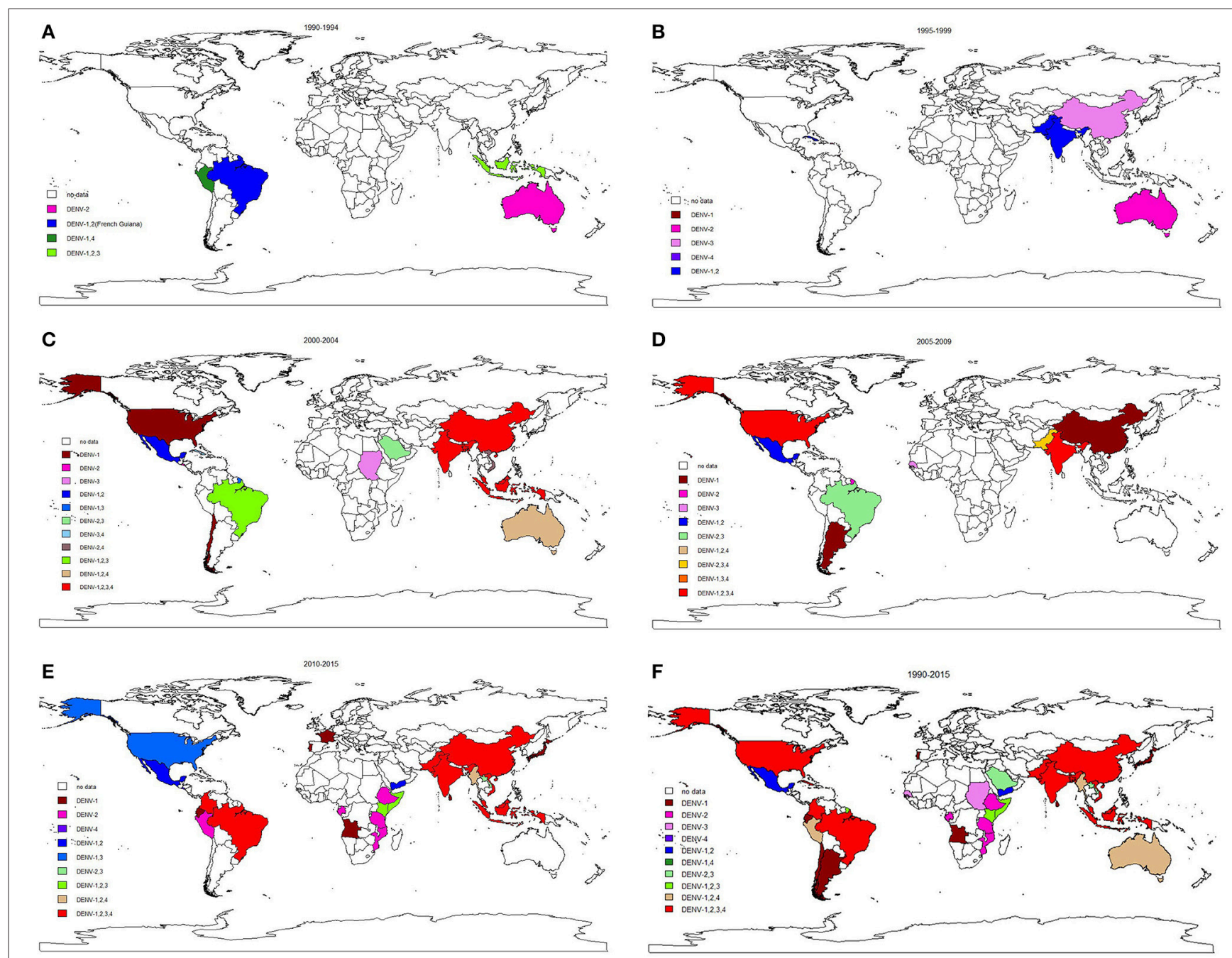

FIGURE 5 | Global serotypes distribution in dengue outbreaks from 1990-2015.

tend to be mild and short (Issack et al., 2010). Wang T. et al. (2015), Wang S. F. et al. (2015), Kim Lien et al. (2015), Kunwar and Prakash (2015), Barde et al. (2015) had reported that rainfall with the hot weather contributed to the dramatically increase of mosquitos. Rainfall provides breeding sites and stimulates egg hatching for dengue transmission vectors and temperature affects the vector's survival and their rate of development and reproduction (Johansson et al., 2009). Chepkorir et al. (2014) and Watts et al. (1987) have demonstrated a significantly higher infection rate of dengue virus at high temperatures for Ae. aegypti mosquitoes, suggesting a potentially significant role of temperature in the dynamics of dengue transmission. It may be due to that the high temperature increases virus reproduction to high titers and reduces the extrinsic incubation period for the dengue virus to be established within the vector, thus leads to the upsurge of dengue outbreaks (Lambrechts et al., 2011). Besides the traditional methods to control vectors, a potential method has been developed during the last decade; scientists released A. aegypti or A. albopictus mosquitoes infected with Wolbachia, which might hinder the insects' ability to transmit the dengue virus and cannot infect humans (Hoffmann et al., 2011; Callaway, 2016).

The results of our study suggested that DENV-2 was the predominant serotype in dengue outbreaks that occurred before 2000, but DENV-3 was the predominant serotype between 2000 and 2009. After 2010, DENV-1 dominated global dengue outbreaks, and DENV-4 was the least frequently identified serotype. We would like to note that at least two serotypes were identified in nearly half the outbreaks, as previous exposure to a single virus could not provide immunity against potential infections with other serotypes (Rahim and Sikder, 2005). Cross-reactive and non-neutralizing antibodies from the primary infection can also bind to the new DENV serotype and facilitate virus entry into susceptible cells. It was a phenomenon known as antibody-dependent enhancement of infection (ADE), considered as the most rational explanation for severe dengue (Simmons et al., 2007; Wang Y. et al., 2015). This can also explain the significantly association 
between secondary infection and DHF in our study (OR: 1.86, 95\% CI: 1.46-2.37). Additionally, we found that the pooled mortality rate was highest in DENV-2 dominated outbreaks $(2.0 \%)$, with significantly higher mortality rates identified in association with this than other serotypes. Although vaccines or therapeutics for dengue are not available, Liu et al. previously identified multiple mosquito galactose specific C-type lectins (mosGCTLs) that facilitated dengue infection; these mosGCTLs were induced in the tissues of mosquitoes with DENV-2 and directly interacted with the DENV-2 surface envelope (E) protein and virions in vitro and in vivo. Membrane blood feeding of antisera against mosGCTLs has been found to efficiently reduce DENV-2 infections among mosquitoes, suggesting that immunization against mosGCTLs may serve as a feasible approach for preventing dengue infection (Liu et al., 2014). Additionally, to reduce the rate of mortality in dengue patients, the application of early clinical and laboratory diagnosis, intravenous rehydration, staff training and hospital reorganization should be ensured during outbreaks (Lo et al., 2007; WHO, 2012).

Dengue infections were characterized by fever (98.1\%), malaise (76.0\%), headache (75.7\%), and asthenia (74.3\%); however, in some cases, bleeding (25.8\%), plasma leakage (8.3\%) and organ impairment (e.g., hepatosplenomegaly, $17.5 \%$ ) were observed. Although dengue was mostly mild and selflimiting in this study, when cases were misdiagnosed by untrained physicians, there may be sufficient time for virus transmission and outbreak development (Huang Xue et al., 2014). In addition to the common symptoms and signs of dengue, manifestations including bleeding (OR: 9.57), pleural effusion (OR: 12.44), and ascites (OR: 13.91) were frequently identified in DHF patients. Thus, physicians should monitor patients for hemorrhage manifestations and capillary leakage, which might indicate the onset of DHF or DSS (Trofa et al., 1997). The results of our study showed that the rates of DHF, secondary infection and mortality in dengue outbreaks were significantly lower in outbreaks occurring after 2010 than in outbreaks before 2010, which suggests that the severity of global dengue infection has somewhat decreased after 2010. Through the use of timely, appropriate clinical management, global outbreak surveillance and sustainable vector control, a great deal of effort has been devoted to achieving the goal set by the World Health Organization in 2012; however, these efforts still face a challenging situation (WHO, 2012).

We believe it is important to note that the age of dengue patients after 2010 was significantly older than that of dengue patients before 2010 (mean pooled age: 34.0 vs. 27.2). Possible reasons for this finding may be that younger people now spend more daytime in enclosed air-conditioned environments and are, therefore, less likely to be exposed to mosquitoes (Lin et al., 2012) and that older people with chronic diseases now visit the doctor more often, causing existing dengue infection to be more likely to be detected (Lee et al., 2006).
There are still some limitations to our study. First, since our study and analysis were based on published articles, some dengue outbreaks may not have been recorded; thus, the result of our study should be interpreted with caution. Second, dengue is a self-limiting disease and most infections are asymptomatic (Bordignon et al., 2008); therefore, a portion of dengue patients might go unidentified during dengue outbreaks. The actual numbers of cases associated with these outbreaks were likely considerably higher than recorded. Regardless of these limitations, we believe that our systematic review and meta-analysis provide useful information regarding the global epidemiology of dengue outbreaks. Our study indicated the countries and WHO regions most seriously affected by dengue outbreaks; the risk factors for and clinical characteristics of dengue infection, DHF and mortality; and the global dengue serotype distribution, all of which could be extremely valuable when targeting prevention and early identification efforts in dengue outbreaks worldwide.

\section{AUTHOR CONTRIBUTIONS}

GY and CJ contributed to the design of the study. CG and ZZ were involved in data acquisition. The data was then analyzed and interpreted by all authors. CG, ZZ, ZW, and YL wrote the first manuscript. GY and CJ critically revised the manuscript for important intellectual content. All authors approved the final version to be submitted.

\section{FUNDING}

This work was supported in part by Training Program of the Major Research Plan of the National Natural Science Foundation of China (Grant numbers: 91543132), National Natural Science Foundation of China (Grant numbers: 81541070, 81101267, and 30901249), Guangdong Natural Science Foundation (Grant numbers: 10151063201000036, S2011010002526, and 2016A030313089), Guangdong Province Medical Research Foundation (Grant number: A2014374 and A2015310) and Project from Jinan university (Grant number: 21612426, 21615426, JNUPHPM2016001, and JNUPHPM2016002), State Key Laboratory for Infectious Disease Prevention and Control (2015SKLID505).

\section{ACKNOWLEDGMENTS}

We would like to thank Hoi Leng Ip, Ka Yu Fu, Mei Leng Ieong, and Chong Iok Lei from Jinan University for providing precious ideas and thoughts.

\section{SUPPLEMENTARY MATERIAL}

The Supplementary Material for this article can be found online at: http://journal.frontiersin.org/article/10.3389/fcimb. 2017.00317/full\#supplementary-material 


\section{REFERENCES}

Amarasinghe, A., Kuritsk, J. N., Letson, G. W., and Margolis, H. S. (2011). Dengue virus infection in Africa. Emerg. Infect. Dis. 17, 1349-1354. doi: 10.3201/eid1708.101515

Barcelos, F. L. (2014). Dengue virus 2 American-Asian Genotype identified during the 2006/2007 outbreak in Piaui, Brazil reveals a Caribbean route of introduction and dissemination of dengue virus in Brazil (vol 9, e104516, 2014). PLoS ONE 9:516. doi: 10.1371/journal.pone.0104516

Barde, P. V., Shukla, M. K., Kori, B. K., Chand, G., Jain, L., Varun, B. M., et al. (2015). Emergence of dengue in tribal villages of Mandla district, Madhya Pradesh, India. Indian J. Med. Res. 141, 584-590. doi: 10.4103/0971-5916. 159517

Bordignon, J., Probst, C. M., Mosimann, A. L., Pavoni, D. P., Stella, V., Buck, G. A., et al. (2008). Expression profile of interferon stimulated genes in central nervous system of mice infected with dengue virus Type-1. Virology 377, 319-329. doi: 10.1016/j.virol.2008.04.033

Callaway, E. (2016). Rio fights Zika with biggest release yet of bacteria-infected mosquitoes. Nature 539, 17-18. doi: 10.1038/nature.2016.20878

Cattand, P., Desjeux, P., Guzman, M. G., Jannin, J., Kroeger, A., Medici, A., et al. (2006). "Tropical diseases lacking adequate control measures: dengue, leishmaniasis, and african trypanosomiasis," in Disease Control Priorities in Developing Countries, 2nd Edn., eds D. T. Jamison, J. G. Breman, A. R. Measham, G. Alleyne, M. Claeson, D. B. Evans, P. Jha, A. Mills, and P. Musgrove (Washington, DC: Oxford University Press) 451-466.

Chang, M. S., Hii, J., Buttner, P., and Mansoor, F. (1997). Changes in abundance and behaviour of vector mosquitoes induced by land use during the development of an oil palm plantation in Sarawak. Trans. R. Soc. Trop. Med. Hyg. 91, 382-386. doi: 10.1016/S0035-9203(97)90248-0

Cheah, W. L., Chang, M. S., and Wang, Y. C. (2006). Spatial, environmental and entomological risk factors analysis on a rural dengue outbreak in Lundu District in Sarawak, Malaysia. Trop. Biomed. 23, 85-96. doi: 10.1038/nature.2016.20878

Chepkorir, E., Lutomiah, J., Mutisya, J., Mulwa, F., Limbaso, K., Orindi, B., et al. (2014). Vector competence of Aedes aegypti populations from Kilifi and Nairobi for dengue 2 virus and the influence of temperature. Parasit. Vectors 7:435. doi: 10.1186/1756-3305-7-435

Figueiredo, L. T. (2007). Emergent arboviruses in Brazil. Rev. Soc. Bras. Med. Trop. 40, 224-229. doi: 10.1590/S0037-86822007000200016

Gubler, D. J. (2011). Dengue, urbanization and globalization: the unholy trinity of the 21(st) century. Trop. Med. Health 39, 3-11. doi: 10.2149/tmh.2011-S05

Guo, X., Yang, H., Wu, C., Jiang, J., Fan, J., Li, H., et al. (2015). Molecular characterization and viral origin of the first dengue outbreak in Xishuangbanna, Yunnan Province, China. (2013). Am. J. Trop. Med. Hyg. 93, 390-393. doi: 10.4269/ajtmh.14-0044

Guzman, M. G., and Harris, E. (2015). Dengue. Lancet 385, 453-465. doi: 10.1016/S0140-6736(14)60572-9

Handoll, H. H. (2006). Systematic reviews on rehabilitation interventions. Arch. Phys. Med. Rehabil. 87:875. doi: 10.1016/j.apmr.2006.04.006

Higgins, J. P., and Thompson, S. G. (2002). Quantifying heterogeneity in a meta-analysis. Stat. Med. 21, 1539-1558. doi: 10.1002/sim.1186

Hoffmann, A. A., Montgomery, B. L., Popovici, J., Iturbe-Ormaetxe, I., Johnson, P. H., Muzzi, F., et al. (2011). Successful establishment of Wolbachia in Aedes populations to suppress dengue transmission. Nature 476, 454-457. doi: 10.1038 /nature10356

Hozo, S. P., Djulbegovic, B., and Hozo, I. (2005). Estimating the mean and variance from the median, range, and the size of a sample. BMC Med. Res. Methodol. 5:13. doi: 10.1186/1471-2288-5-13

Huang Xue, Y., Ma Hong, X., Wang Hai, F., Du Yan, H., Su, J., Li Xing, L., et al. (2014). Outbreak of dengue fever in Central China. (2013). Biomed. Environ. Sci. 27, 894-897. doi: 10.3967/bes2014.125

Ishak, H., Miyagi, I., Toma, T., and Kamimura, K. (1997). Breeding habitats of Aedes aegypti (L) and Aedes. albopictus (Skuse) in villages of Barru, South Sulawesi, Indonesia. Southeast. Asian J. Trop. Med. Public Health 28, 844-850.

Issack, M. I., Pursem, V. N., Barkham, T. M. S., Ng, L. C., Inoue, M., and Manraj, S. S. (2010). Reemergence of dengue in Mauritius. Emerg. Infect. Dis. 16, 716-718. doi: 10.3201/eid1604.091582

Jansen, C. C., and Beebe, N. W. (2010). The dengue vector Aedes aegypti: what comes next. Microbes Infect. 12, 272-279. doi: 10.1016/j.micinf.2009.12.011
Johansson, M. A., Cummings, D. A., and Glass, G. E. (2009). Multiyear climate variability and dengue-El Nino southern oscillation, weather, and dengue incidence in Puerto Rico, Mexico, and Thailand: a longitudinal data analysis. PLoS Med. 6:e1000168. doi: 10.1371/journal.pmed.1000168

Kim Lien, P. T., Duoc, V. T., Gavotte, L., Cornillot, E., Nga, P. T., Briant, L., et al. (2015). Role of Aedes aegypti and Aedes albopictus during the 2011 dengue fever epidemics in Hanoi, Vietnam. Asian Pac. J. Trop. Med. 8, 543-548. doi: 10.1016/j.apjtm.2015.06.009

Kunwar, R., and Prakash, R. (2015). Dengue outbreak in a large military station: have we learnt any lesson? Med. J. Armed. Forces India 71, 11-14. doi: 10.1016/j.mjafi.2014.11.002

Lambrechts, L., Paaijmans, K. P., Fansiri, T., Carrington, L. B., Kramer, L. D., Thomas, M. B., et al. (2011). Impact of daily temperature fluctuations on dengue virus transmission by Aedes aegypti. Proc. Natl. Acad. Sci. U.S.A. 108, 7460-7465. doi: 10.1073/pnas.1101377108

Lancet, T. (2013). Dengue-an infectious disease of staggering proportions. Lancet 381, 2136. doi: 10.1016/s0140-6736(13)61423-3

Lee, M. S., Hwang, K. P., Chen, T. C., Lu, P. L., and Chen, T. P. (2006). Clinical characteristics of dengue and dengue hemorrhagic fever in a medical center of southern Taiwan during the 2002 epidemic. J. Microbiol. Immunol. Infect. 39, 121-129.

Lin, C. H., Schioler, K. L., Jepsen, M. R., Ho, C. K., Li, S. H., and Konradsen, F. (2012). Dengue outbreaks in high-income area, Kaohsiung City, Taiwan, 20032009. Emerg. Infect. Dis. 18, 1603-1611. doi: 10.3201/eid1810.111929

Liu, Y., Zhang, F., Liu, J., Xiao, X., Zhang, S., Qin, C., et al. (2014). Transmissionblocking antibodies against mosquito C-type lectins for dengue prevention. PLoS Pathog 10:e1003931. doi: 10.1371/journal.ppat.1003931

Lo, C. L., Yip, S. P., Cheng, P. K., To, T. S., Lim, W. W., and Leung, P. H. (2007). One-step rapid reverse transcription-PCR assay for detecting and typing dengue viruses with GC tail and induced fluorescence resonance energy transfer techniques for melting temperature and color multiplexing. Clin. Chem. 53, 594-599. doi: 10.1373/clinchem.2006.077446

Organization, W. H. (2016). Dengue and Severe Dengue. Available online at: http://www.who.int/mediacentre/factsheets/fs117/en/ (Accessed 24 Mar 2016).

Rahim, M. A., and Sikder, M. S. (2005). Clinicopathologic manifestations and outcome of dengue fever and dengue haemorrhagic fever. Bangladesh Med. Res. Counc. Bull. 31, 36-45.

Saswat, T., Kumar, A., Kumar, S., Mamidi, P., Muduli, S., Debata, N. K., et al. (2015). High rates of co-infection of Dengue and Chikungunya virus in Odisha and Maharashtra, India during 2013. Infect. Genet. Evol. 35, 134-141. doi: 10.1016/j.meegid.2015.08.006

Screaton, G., Mongkolsapaya, J., Yacoub, S., and Roberts, C. (2015). New insights into the immunopathology and control of dengue virus infection. Nat. Rev. Immunol. 15, 745-759. doi: 10.1038/nri3916

Simmons, C. P., Chau, T. N., Thuy, T. T., Tuan, N. M., Hoang, D. M., Thien, N. T., et al. (2007). Maternal antibody and viral factors in the pathogenesis of dengue virus in infants. J. Infect. Dis. 196, 416-424. doi: 10.1086/519170

Simmons, C. P., Farrar, J. J., Nguyen v, V., and Wills, B. (2012). Dengue. N. Engl. J. Med. 366, 1423-1432. doi: 10.1056/NEJMra1110265

Succo, T., Leparc-Goffart, I., Ferre, J., Roiz, D., Broche, B., Maquart, M., et al. (2016). Autochthonous dengue outbreak in Nimes, South of France, July to September 2015. Eurosurveillance 21, 5-11. doi: 10.2807/1560-7917.es.2016.21.21.30240

Trofa, A. F., DeFraites, R. F., Smoak, B. L., Kanesa-thasan, N., King, A. D., Burrous, J. M., et al. (1997). Dengue fever in US military personnel in Haiti. JAMA 277, 1546-1548. doi: 10.1001/jama.1997.03540430058033

Wang, B., Yang, H., Feng, Y., Zhou, H., Dai, J., Hu, Y., et al. (2016). The distinct distribution and phylogenetic characteristics of dengue virus serotypes/genotypes during the 2013 outbreak in Yunnan, China: phylogenetic characteristics of 2013 dengue outbreak in Yunnan, China. Infect. Genet. Evol. 37, 1-7. doi: 10.1016/j.meegid.2015.10.022

Wang, S. F., Chang, K., Lu, R. W., Wang, W. H., Chen, Y. H., Chen, M., et al. (2015). Large dengue virus type 1 outbreak in Taiwan. Emerg. Microbes. Infect. 4:e46. doi: 10.1038/emi.2015.46

Wang, T., Wang, M., Shu, B., Chen, X. Q., Luo, L., Wang, J. Y., et al. (2015). Evaluation of inapparent dengue infections during an outbreak in Southern China. PLoS. Negl. Trop. Dis. 9:e0003677. doi: 10.1371/journal.pntd.00 03677 
Wang, Y., Si, L., Luo, Y., Guo, X., Zhou, J., Fang, D., et al. (2015). Replacement of pr gene with Japanese encephalitis virus pr using reverse genetics reduces antibody-dependent enhancement of dengue virus 2 infection. Appl. Microbiol. Biotechnol. 99, 9685-9698. doi: 10.1007/s00253-0156819-3

Watts, D. M., Burke, D. S., Harrison, B. A., Whitmire, R. E., and Nisalak, A. (1987). Effect of temperature on the vector efficiency of Aedes aegypti for dengue 2 virus. Am. J. Trop. Med. Hyg. 36, 143-152. doi: 10.4269/ajtmh.1987. 36.143

Whitehorn, J., Duong Thi Hue, K., Nguyet Minh, N., Nguyen, H. L., Kyrylos, P. P., Carrington, L. B., et al. (2015). Comparative susceptibility of Aedes albopictus and Aedes aegypti to dengue virus infection after feeding on blood of viremic humans: implications for public health. J. Infect. Dis. 212, 1182-1190. doi: 10.1093/infdis/jiv173

WHO (2012). Global Strategy for Dengue Prevention and Control, 2012-2020. Geneva: World Health Organization.

WHO/TDR (2009). Dengue Guidelines for Diagnosis, Treatment, Prevention and Control. New Edition. Geneva: World Health Organization.
Wilder-Smith, A., Quam, M., Sessions, O., Rocklov, J., Liu-Helmersson, J., Franco L., et al. (2014). The 2012 dengue outbreak in Madeira: exploring the origins. Euro. Surveill. 19:20718. doi: 10.2807/1560-7917.ES2014.19.8.20718

Wu, W., Bai, Z., Zhou, H., Tu, Z., Fang, M., Tang, B., et al. (2011). Molecular epidemiology of dengue viruses in southern China from 1978 to 2006. Virol. J. 8:322. doi: $10.1186 / 1743-422 \mathrm{X}-8-322$

Conflict of Interest Statement: The authors declare that the research was conducted in the absence of any commercial or financial relationships that could be construed as a potential conflict of interest.

Copyright (C) 2017 Guo, Zhou, Wen, Liu, Zeng, Xiao, Ou, Han, Huang, Liu, Ye, Zou, Wu, Wang, Zeng, Jing and Yang. This is an open-access article distributed under the terms of the Creative Commons Attribution License (CC BY). The use, distribution or reproduction in other forums is permitted, provided the original author(s) or licensor are credited and that the original publication in this journal is cited, in accordance with accepted academic practice. No use, distribution or reproduction is permitted which does not comply with these terms. 